\title{
On Quasi-Linear Parabolic Equations of the Second Order
}

\section{AVNER FRIEDMAN}

\author{
Communicated by E. HopF
}

Introduction. Let $D$ denote a bounded $(n+1)$-dimensional domain and let its points be denoted by $(x, t)=\left(x_{1}, \cdots, x_{n}, t\right)$. Let $D$ be bounded by two hyperplanes $t=0$ and $t=t_{0}>0$, and by a surface $C$ ( $C$ is a closed set) lying between these hyperplanes. Denote by $\bar{D}$ the closure of $D$, by $B$ the basis of $D$ $(B=\bar{D} \cap\{t=0\})$, by $D_{\tau}$ the subdomain $D \cap\{0<t<\tau\}$, by $C_{\tau}$ the intersestion $C \cap\{0 \leqq t \leqq \tau\}$ and, finally, denote by $\partial D_{\tau}(\partial D)$ the set $C_{\tau} \cup B(C \cup B)$. Consider the linear operator

$$
L u \equiv \sum_{i, j=1}^{n} a_{i j}(x, t) \frac{\partial^{2} u}{\partial x_{i} \partial x_{i}}+\sum_{i=1}^{n} b_{i}(x, t) \frac{\partial u}{\partial x_{i}}+c(x, t) u-\frac{\partial u}{\partial t}
$$

with coefficients defined in $\bar{D}$. In Section 1 and occasionally in Section 2 we shall assume that the coefficients of $L$ satisfy the following assumptions:

(A) The functions $a_{i j}$ have continuous derivatives up to the fifth order with respect to $(x, t)$ in $\bar{D}$; the functions $b_{i}$ and $c$ have continuous derivatives up to the third order with respect to $(x, t)$ in $\bar{D}$.

(B) $L$ is of parabolic type, that is, there exists a positive constant $H$ such that for every point $(x, t)$ in $\bar{D}$ and for every real vector $\xi=\left(\xi_{1}, \cdots, \xi_{n}\right)$

$$
\sum_{i, j=1}^{n} a_{i j}(x, t) \xi_{i} \xi_{i} \geqq H \sum_{i=1}^{n} \xi_{i}^{2}
$$

Throughout Section 1 and occasionally in Section 2 we shall assume that the surface $C$ satisfies the following property:

$\left(\mathrm{E}^{\prime}\right)$ To every point $P$ of $C$ there exists an $(n+1)$-dimensional sphere $V$ with center $P$ such that the intersection $C \cap V$ can be represented, for some $i(1 \leqq i \leqq n)$, in the form

$$
x_{i}=h\left(x_{1}, \cdots, x_{i-1}, x_{i+1}, \cdots, x_{n}, t\right)
$$

where $h$ has continuous derivatives up to the sixth order in all its arguments.

Prepared under ONR Contract Nont-908(09) NR 041037 with Indiana University. 
Let $f=f(x, t, u, \nabla u)$ (where $\left.\nabla u=\left(u_{x_{1}}, \cdots, u_{x_{n}}\right)\right)$ be defined in the cylinder

$$
(x, t) \varepsilon \bar{D}, \quad-\infty<u<\infty, \quad-\infty<u_{x_{i}}<\infty \quad(i=1, \cdots, n),
$$

and consider the quasi-linear equation

$$
L u=f(x, t, u, \nabla u)
$$

with the Dirichlet data

$$
u(x, 0)=g(x) \quad \text { on } B, \quad u(x, t)=k(x, t) \quad \text { on } \quad C .
$$

In this paper we consider the problems of existence and uniqueness of solutions of the Dirichlet problem (4), (5). An existence theorem for $n=1, L u=u_{x x}-u_{t}$ and $D$ a rectangle was proved by Ciliberto [2], [3] under the condition

$$
|f(x, t, u, w)| \leqq A+B(|u|+|w|) \quad(A>0, B>0)
$$

provided the height of the rectangle $D$ is sufficiently small (depending on the constants $A, B)$. He used the method of integral equations with a fundamental solution as a kernel. More recently SATō [11] has considered existence and uniqueness problems for weak solutions of the Dirichlet problem corresponding to the elliptic equation $\Delta u=f\left(x, y, u, u_{x}, u_{y}\right)$. His methods were extended by PINI [10] to parabolic equations of the form $u_{x x}-u_{t}=f\left(x, t, u, u_{x}\right)$. In particular, he proved the existence of weak solutions provided $f$ satisfies the inequality

$$
|f(x, t, u, w)| \leqq A+B|u|^{\lambda} \quad(0 \leqq \lambda<1, A>0, B>0) .
$$

Both papers use the following well known idea: With the aid of Green's function in the whole domain one defines a transformation $v=T u$ whose fixed points are the desired weak solutions; then one shows that a general fixed point theorem can be applied to the transformation $T$.

In this paper we define the transformation $v=T u$ in a different manner: $v$ is a solution of the equation $L v=f(x, t, u, \nabla u)$ with the appropriate boundary values. Use is made of an existence theorem which we derived in an earlier paper [7]. We also make use of Green's function for rectangular domains constructed in [8].

The results of this paper contain all those mentioned above as very special cases. In $\$ 1$ we prove an existence theorem; use is made of ScHAUdER's fixed point theorem [12]. In $\$ 2$ we use the methods of [10], [11] to establish, by simple comparison considerations, a few uniqueness properties. We, further, restate the existence theorem of $\$ 1$ in a different form. In $\S 3$ we mention that the results of $\$ \$ 1$ and 2 can be extended to the case of second order elliptic equations.

We finally remark that existence and stability theorems for systems of the form (4), (5) in semi-infinite cylinders were proved for "small" $f$ (for instance, $|f(x, t, u)| \leqq \lambda|u|$ and $\lambda$ is small) by various authors (for a complete treatment see [8]). 


\section{An Existence Theorem}

1.1. The transformation $v=T u$. Throughout this section it is assumed that $f$ satisfies a Hölder condition in compact subsets of the cylinder (3) and that (A), (B), ( $\left.\mathrm{E}^{\prime}\right)$ hold. For any $\alpha(0<\alpha<1)$ denote by $C_{1+\alpha}$ the set of functions $u(x, t)$ defined and Hölder continuous (exponent $\alpha$ ) in the sense of [7] together with their first $x$-derivatives in $\bar{D}$, and define in $C_{1+\alpha}$ a norm

$$
|u|_{1+\alpha}=\operatorname{l.u.b.}_{(x, t)_{\varepsilon} D}\left\{|u(x, t)|+\sum_{j=1}^{n}\left|\frac{\partial}{\partial x_{j}} u(x, t)\right|\right\}+H[u]+\sum_{j=1}^{n} H\left[\frac{\partial u}{\partial x_{j}}\right]
$$

where

$$
H[v]=\operatorname{liu}_{P, Q \mathrm{e} D} \frac{|v(P)-v(Q)|}{d(P, Q)^{\alpha}}
$$

is Hölder's coefficient of $v$ (if $P=(x, t), Q=\left(x^{\prime}, t^{\prime}\right)$, then $d(P, Q)=\left(\left|x-x^{\prime}\right|^{2}+\right.$ $\left.\left.\left|t-t^{\prime}\right|\right)^{\frac{1}{2}}\right)$. Similarly define $C_{2+\delta}$ to be the set of function $u(x, t)$ with finite norm defined by

$$
|u|_{2+\delta}=\sum_{i=0}^{2}\left\{\underset{(x, t) \varepsilon D}{\text { l.u.b. }}\left|D_{x}^{i} u(x, t)\right|+H\left[D_{x}^{i} u\right]\right\}+\underset{(x, t)_{\varepsilon D}}{\operatorname{lu}_{(x)} . \mathrm{b} .}\left|D_{t} u(x, t)\right|+H\left[D_{t} u\right]
$$

where $H[v]$ in (1.3) is defined as in (1.2) but with $\alpha$ replaced by $\delta$. In (1.3) $D_{x}^{i} u$ denotes any $i^{\text {th }}$ partial derivative of $u$; the summation is understood to be taken also on all the $i^{\text {th }}$ partial derivatives of $u$. Note that $C_{1+\alpha}$ and $C_{2+\delta}$ are Banach spaces; bounded closed sets in $C_{1+\beta}$ are compact in $C_{1+\alpha}$ whenever $\alpha<\beta<1$.

For any $M>0$ define a set $C_{M}$ in the following way:

$$
C_{M}=\left\{\left.u|| u\right|_{1+\alpha} \leqq M\right\} .
$$

For each $u \varepsilon C_{M}$ define a transform $v=T u$ in the following way: $v$ is the solution in $D$ of the equation

$$
L v=f(x, t, u, \nabla u)
$$

which vanishes on the boundary $\partial D=B+C$ of $D$. From [7] we conclude that $v$ exists and is uniquely defined; furthermore, for some positive $\delta$ (depending on $f$ and $M$ but independent of the specific $u$ )

$$
|v|_{2+\delta} \leqq \text { const. }|\tilde{f}|_{\delta} \leqq \text { const. }<\infty
$$

where $\tilde{f}(x, t)=f(x, t, u(x, t), \nabla u(x, t))$. However, since bounded closed sets in $C_{2+\delta}$ need not be compact in $C_{1+\alpha}$ when $\delta<\alpha$, we cannot use (1.6) to prove that the set $\left\{T u \mid u \varepsilon C_{M}\right\}$ is precompact in the norm ||$_{1+\alpha} ;$ a proof of this statement will be given later. We proceed to prove that, under some conditions on $f$, the transformation $T$ maps $C_{M}$ into itself.

1.2. $T$ maps $C_{M}$ into itself. One has to prove that

$$
|T u|_{1+\alpha} \leqq M .
$$


1.2.1. Green's function. To prove (1.7) we denote by ||$_{p}^{D^{\circ}}$ the norm ||$_{p}$ taken with respect to the domain $D^{0}$ instead of $D$. Let $D^{0}$ be an $(n+1)$-dimensional rectangle contained in $D$ all of whose edges are parallel to the coordinate axes. In [8] we constructed Green's function $G(x, t ; \xi, \tau)$ corresponding to $L^{*}$, the adjoint of $L$, provided $L$ satisfied the assumption (B) and an assumption much weaker than (A). $G(x, t ; \xi, \tau)$ is defined for $(x, t)$ and $(\xi, \tau)$ in $D^{0}$, $t<\tau$; vanishes on the lateral boundary $C^{0}$ of $D^{0}$; satisfies for every fixed $(\xi, \tau)$ the equation $L^{*} G=0$, and for every fixed $(x, t)$ the equation $L G=0$; satisfies the relation

$$
G(x, t ; \xi, \tau)=G^{*}(\xi, \tau ; x, t)
$$

where $G^{*}$ is Green's function corresponding to $L$ in $D^{0}$; finally, when $\tau \searrow t G$ behaves "essentially" like

$$
\frac{1}{(\tau-t)^{\frac{1}{3} n}} \exp \left\{-\frac{\sum A_{i j}(x, t)\left(x_{i}-\xi_{i}\right)\left(x_{j}-\xi_{i}\right)}{4(\tau-t)}\right\}
$$

where $\left(A_{i j}(x, t)\right)$ is the matrix inverse to $\left(a_{i j}(x, t)\right)$.

In what follows we shall need the following inequalities:

$$
\begin{gathered}
\left|D_{\xi}^{i} D_{x}^{i} G(x, t ; \xi, \tau)\right| \leqq \frac{A_{0}}{(\tau-t)^{\frac{1}{2}(n+i+j)}} \exp \left\{-\frac{A|x-\xi|^{2}}{\tau-t}\right\} \\
\equiv A_{0} V_{n+i+j}(x, t ; \xi, \tau) \quad(i, j=0,1,2), \\
\left|D_{\xi}^{i} D_{x}^{i} D_{\tau} G(x, t ; \xi, \tau)\right| \leqq A_{0} V_{n+i+i+2} \quad(i, j=0,1) ;
\end{gathered}
$$

here $A, A_{0}$ are positive constants. In the sequel $A_{i}$ will be used to denote appropriate constants.

Proof of (1.10), (1.11). The proofs of (1.10) for $i, j=0,1$ and of (1.11) for $i=j=0$ follow from the explicit form of $G$ (in [8]). From the equation

$$
\frac{\partial G}{\partial \tau}=\left(L^{*}-\frac{\partial}{\partial \tau}\right) G
$$

$\left(L^{*}-\partial / \partial \tau\right.$ involves only $\xi$-derivatives of the first two orders) we conclude that the proof of (1.11) will be completed if we show that

$$
\left|D_{x}^{i} D_{\xi}^{i} G(x, t ; \xi, \tau)\right| \leqq A_{0} V_{n+i+j}(x, t ; \xi, \tau) \quad(i+j=3,4) .
$$

From the explicit formula of $G$ as a convergent series of fundamental solutions [8] and from the method of EIDELMAN [5] to prove differentiability of fundamental solutions (in particular, [5; formulas $\left.\left(2.14_{m}\right),(2.39)\right]$ ) we conclude that the assumptions (A), (B) are sufficient to ensure the validity of (1.12).

1.2.2. Estimation of $T u$ in the interior. For simplicity we take $D^{0}$ to be a semi-cube with edge $d$ and top $P^{0}=\left(x^{0}, t^{0}\right)$, that is, $D^{0}$ is defined by $-d<x_{i}-x_{i}^{0}<d \quad(i=1, \cdots, n), d^{2}<t-t^{0}<0 \quad\left(x^{0}=\left(x_{1}^{0}, \cdots, x_{n}^{0}\right)\right)$. 
We assume that $\bar{D}^{0}$, the closure of $D^{0}$, is contained in $\bar{D}-\partial D$. Denote by $\varphi(x, t)$ an infinitely differentiable function which is equal to 1 in $\bar{D}_{1}$ and vanishes outside of $\bar{D}_{\frac{3}{2}}$; here $D_{\lambda}(\lambda>0)$ denotes the semi-cube with edge $\lambda d$ and top $P^{0}$. If we use Green's identity with $v(x, t)$ and $\varphi(x, t) G(x, t ; \xi, \tau)$ where

$$
L v(x, t)=f(x, t, u(x, t), \nabla u(x, t)) \equiv \tilde{f}(x, t),
$$

then we obtain, as in [6], the representation

$$
\begin{aligned}
v(\xi, \tau)=\int_{t^{\prime}}^{\tau} & \int_{B^{\circ}} \varphi(x, t) \tilde{f}(x, t) G(x, t ; \xi, \tau) d x d t \\
& \quad-\int_{t^{\prime}}^{\tau} \int_{B^{\circ}} v(x, t) L^{*}[\varphi(x, t) G(x, t ; \xi, \tau)] d x d t \equiv H_{0}-J_{0}
\end{aligned}
$$

where $t^{\prime}=t^{0}-d^{2}, B^{0}$ is the basis of $D^{0}$, and where $H_{0}$ and $J_{0}$ denote the first and second integrals respectively; (1.14) is valid provided the distance $\left\{\left|\xi-x^{0}\right|^{2}+\left|\tau-t^{0}\right|\right\}^{3}$ from $(\xi, \tau)$ to $P^{0}$ is less than $\frac{1}{2} d$.

We note that $L^{*}[\varphi G]$ (in $J_{0}$ ) vanishes on the sets $\bar{D}_{1}$ and $D^{0}-\bar{D}_{\frac{3}{q}}$; hence the integration in $J_{0}$ has to be taken only on the set $\bar{D}_{\frac{1}{3}}-\bar{D}_{\frac{1}{2}}$. Using the fact that $D_{\xi}^{2} D_{x} G, D_{\tau} D_{x} G$ and $D_{\xi} D_{\tau} D_{x} G$ are continuous for $(x, t) \neq(\xi, \tau)$, we easily find that

$$
\left|J_{0}\right|_{1+\alpha}^{D_{1} / \alpha} \leqq A_{1} \operatorname{liu}_{D^{\circ}} . \text { b. }|v| .
$$

We proceed to prove that

$$
\left|H_{0}\right|_{1+\alpha}^{D^{\circ}} \leqq A_{2} \text { l.u.b. } \mid \tilde{D^{\circ}} \text {. }|\tilde{f}| \text {. }
$$

1.2.3. Continuation. Proof of (1.16). It will be enough to prove that

$$
\left|D_{\xi} H_{0}(\xi, \tau)-D_{\xi^{\prime}} H_{0}\left(\xi^{\prime}, \tau^{\prime}\right)\right| \leqq A_{3}\left(1 .{ }_{D^{\circ}} \text {.b. }|\tilde{f}|\right)\left\{\left|\xi-\xi^{\prime}\right|^{\alpha}+\left|\tau-\tau^{\prime}\right|^{\frac{1}{\alpha} \alpha}\right\} .
$$

We shall prove (1.17) in two special cases: first in case $\tau=\tau^{\prime}$ and then in case $\xi=\xi^{\prime}$; the proof of the general case is then very easily completed.

Case (i): $\tau=\tau^{\prime}$. Writing

$$
D_{\xi} H_{0}(\xi, \tau)=\int_{t^{\prime}}^{\tau} \int_{B^{\circ}} \varphi(x, t) \tilde{f}(x, t) D_{\xi} G(x, t ; \xi, \tau) d x d t
$$

and denoting $h^{*}=(0, \cdots, 0, h, 0, \cdots, 0)$, we see that all we need to prove is that

$$
\int_{t^{\prime}}^{\tau} \int_{B^{\circ}}\left|D_{\xi} G\left(x, t ; \xi+h^{*}, \tau\right)-D_{\xi} G(x, t ; \xi, \tau)\right| d x d t \leqq A_{4} h^{\alpha}
$$

where we assumed (without loss of generality) that $h>0$.

Let $\gamma=h^{2}$ and denote by $D_{\gamma}^{0}$ the domain $D^{0} \cap\{t<\gamma\}$ and by $S_{\gamma}$ the domain $D_{\tau}^{0}-D_{\tau-\gamma}^{0}$ (we assume $\tau-\gamma \geqq t^{\prime}$; otherwise we replace $\tau-\gamma$ by $t^{\prime}$ ). Then, 
substituting (for each fixed $t)|x-\xi|=(\tau-t)^{\frac{1}{2}} \rho$ and using (1.10), we get

$$
\begin{aligned}
\iint_{S_{\gamma}} \mid D_{\xi} G\left(x, t ; \xi+h^{*}, \tau\right) & -D_{\xi} G(x, t ; \xi, \tau) \mid d x d t \\
& \leqq 2 A_{0} \iint_{S_{\gamma}} V_{n+1}(x, t ; \xi, \tau) d x d t \\
\leqq & A_{5} \int_{\tau-\gamma}^{\tau} \int_{0}^{\infty} \frac{e^{-A \rho^{2}} \rho}{\tau-t}(\tau-t)^{\frac{1}{2}} d \rho d t \leqq A_{6} \gamma^{\frac{3}{2}} .
\end{aligned}
$$

To estimate the rest of the integral in (1.19) we shall make use of the formula

$$
D_{\xi} G\left(x, t ; \xi+h^{*}, \tau\right)-D_{\xi} G(x, t ; \xi, \tau)=\int_{\xi}^{\xi+h^{*}} D_{\xi}^{2} G(x, t ; \zeta, \tau) d \zeta
$$

where $\zeta$ varies in the interval connecting $\xi$ with $\xi+h^{*}$. We obtain

$$
\begin{aligned}
& \iint_{D^{0_{\tau}-\gamma}}\left|D_{\xi} G\left(x, t ; \xi+h^{*}, \tau\right)-D_{\xi} G(x, t ; \xi, \tau)\right| d x d t \\
& \quad \leqq \int_{\xi}^{\xi+h^{*}} d \zeta \iint_{D^{0_{r-\gamma}}}\left|D_{\xi}^{2} G(x, t ; \zeta, \tau)\right| d x d t .
\end{aligned}
$$

Using (1.10) we find that the last integral is bounded by

$$
A_{7} \int_{\xi}^{\xi+h^{*}} d \zeta \int_{t^{\prime}}^{\tau-\gamma} \frac{d t}{\tau-t} \leqq A_{8} h \gamma^{-\epsilon}
$$

where $\epsilon$ is an arbitrary positive number $\left(A_{8}\right.$ depends on $\epsilon$ ).

Combining the last result with (1.20) and taking $\gamma=h^{2}, 2 \epsilon<1-\alpha$, the proof of (1.19) is completed.

Case (ii): $\xi=\xi^{\prime}$. Let $\gamma>0, h>0$. Denoting $S_{\gamma}=D_{\tau}^{0}-D_{\tau-\gamma}^{0}, S_{h}^{0}=D_{\tau+h}^{0}-$ $D_{\tau}^{0}$ and using (1.18), we get

$$
\begin{aligned}
& \left|D_{\xi} H_{0}(\xi, \tau+h)-D_{\xi} H_{0}(\xi, \tau)\right| \leqq A_{9}(\text { l.u.b. }|\tilde{f}|) \\
& \cdot\left\{\iint_{D^{0_{\tau}-\gamma}}\left|D_{\xi} G(x, t ; \xi, \tau+h)-D_{\xi} G(x, t ; \xi, \tau)\right| d x d t\right. \\
& +\iint_{S_{\gamma}}\left[\left|D_{\xi} G(x, t ; \xi, \tau+h)\right|+\left|D_{\xi} G(x, t ; \xi, \tau)\right|\right] d x d t \\
& \left.+\iint_{S^{0_{h}}}\left|D_{\xi} G(x, t ; \xi, \tau+h)\right| d x d t\right\}=A_{9}(\text { l.u.b. }|\tilde{f}|)\left(I_{1}+I_{2}+I_{3}\right)
\end{aligned}
$$

where $I_{i}$ denotes the $j^{\text {th }}$ integral on the right side of the inequality. To estimate $I_{3}$ we use (1.10) and then substitute $|x-\xi|=\rho(\tau-t)^{\frac{1}{2}}$. We obtain

$$
I_{3} \leqq A_{10} h^{\frac{1}{2}} \text {. }
$$

Similarly we obtain

$$
I_{2} \leqq A_{11}\left(\gamma^{\frac{1}{2}}+h^{\frac{1}{2}}\right)
$$


It remains to estimate $I_{1}$. If we use the formula

$$
D_{\xi} G(x, t ; \xi, \tau+h)-D_{\xi} G(x, t ; \xi, \tau)=\int_{\tau}^{\tau+h} D_{\xi} D_{\sigma} G(x, t ; \xi, \sigma) d \sigma
$$

and (1.11), then we obtain (on substituting $|x-\xi|=\rho(\sigma-t)^{\frac{1}{2}}$ )

$$
I_{1} \leqq A_{12} h \gamma^{-\frac{1}{2}} \text {. }
$$

Combining (1.25) with (1.24), (1.23) and (1.22), and taking $\gamma=h$, the proof of (1.17), in case $\xi=\xi^{\prime}$, is completed.

Conclusion. Having completed the proof of (1.16) we obtain, on using (1.15) and (1.14), the inequality

$$
|v|_{1+\alpha}^{\bar{D}_{1 / \alpha}} \leqq A_{1} \underset{D^{\circ}}{1 . u . b .}|v|+A_{2} \operatorname{lip}_{D^{\circ}}^{\text {l.b. }}|\tilde{f}| \text {. }
$$

A simple argument shows that for any closed subdomain $D_{1}$ of $\bar{D}-\partial D$ the inequality

$$
|v|_{1+\alpha}^{D_{1}} \leqq A_{13} \operatorname{l.u.b.}_{D}|v|+A_{14} \text { l.u.b. }|\tilde{f}|
$$

holds with constants $A_{13}, A_{14}$ depending on $L$ and $D_{1}$.

1.2.4. Estimation of $T u$ near the boundary. It remains to study the behavior of $T u$ near the boundary $\partial D=B \cup C$ of $D$. Let $P$ be a point on $\partial D$. There are three possibilities: either $P_{\varepsilon} B$ or $P \varepsilon C$ or $P \varepsilon B \cap C$. It will be enough to consider the last case; the first two cases can be treated in the same manner. Let $V$ be an $(n+1)$-dimensional sphere with center $P$ so that $C \cap V$ can be represented in the form (2) with $h$ having six continuous derivatives. For simplicity we assume that $i=n$ in (2).

The transformation

$$
\begin{gathered}
t^{\prime}=t, \quad x_{i}^{\prime}=x_{i} \quad(i=1, \cdots, n-1), \\
x_{n}^{\prime}=x_{n}-h\left(x_{1}, \cdots, x_{n-1}, t\right)
\end{gathered}
$$

maps a certain one-sided closed neighborhood of $C \cap V$, call it $E(E \subset \bar{D})$, onto a semi-cube $E^{\prime}$ with edges parallel to the $x_{i}^{\prime}$ and $t^{\prime}$ axes. Let $E^{\prime}$ be defined by

$$
\begin{gathered}
\left|x_{i}^{\prime}-\beta_{i}\right| \leqq d \quad(i=1, \cdots, n-1) \\
-d<x_{n}^{\prime}<0, \quad 0<t^{\prime}<d^{2} .
\end{gathered}
$$

Equation (1.13) is transformed into $L^{\prime} w=\tilde{f}^{\prime}$ where $w\left(x^{\prime}, t^{\prime}\right)=v(x, t), \tilde{f}^{\prime}\left(x^{\prime}, t^{\prime}\right)=$ $\tilde{f}(x, t)$ and $L^{\prime}$ satisfies in $E^{\prime}$ the assumptions (A), (B) (here we made use of the differentiability properties of $h$ ).

If we prove that

$$
|w|_{1+\alpha}^{E^{\prime \prime}} \leqq A_{15} \operatorname{l.u.b.}_{E^{\prime}}|w|+A_{16} \underset{E^{\prime}}{\operatorname{lou} . \mathrm{b} .}\left|\tilde{f}^{\prime}\right|
$$


where $E^{\prime \prime}$ is the rectangle

$$
\begin{gathered}
\left|x_{i}^{\prime}-\beta_{i}\right| \leqq \frac{1}{2} d \quad(i=1, \cdots, n-1), \\
-\frac{1}{2} d<x_{n}^{\prime}<0, \quad 0<t^{\prime}<d^{2}
\end{gathered}
$$

then it will easily follow that

$$
|v|_{1+\alpha}^{E_{0}} \leqq A_{17} \operatorname{low}_{E} \text {.b. }|v|+A_{18} \text { l.u.b. }_{E}|\tilde{f}|
$$

where $E^{\prime \prime}$ is the image of $E_{0}$ under the transformation inverse to (1.28). Combining (1.31) with (1.27) and using the Heine-Borel theorem we conclude that

$$
|v|_{1+\alpha}^{D} \leqq A_{19} \underset{D}{\text { l.u.b. }}|v|+A_{20} \text { l.u.b. }|\tilde{f}| \text {. }
$$

Proof of (1.29). Let $G^{\prime}\left(x, t^{\prime} ; \xi^{\prime}, \tau^{\prime}\right)$ be Green's function corresponding to the adjoint of $L^{\prime}$ in $E^{\prime}$ and write Green's identity with $w\left(x^{\prime}, t^{\prime}\right)$ and $G^{\prime}\left(x^{\prime}, t^{\prime} ; \xi^{\prime}, \tau^{\prime}\right)$ (not $\left.\varphi G^{\prime} !\right)$. Noting that $G^{\prime}$ vanishes on the lateral boundary of $E^{\prime}$ and that $w$ vanishes on the faces $x_{n}^{\prime}=0$ and $t^{\prime}=0$ of $E^{\prime}$, we obtain a representation analogous to (1.14) with one important difference: $J_{0}$ does not appear and, on the other hand, there appear boundary integrals of the form

$$
\int_{S_{i}} g_{i}\left[\frac{\partial}{\partial x^{\prime}} G^{\prime}\left(x^{\prime}, t^{\prime} ; \xi^{\prime}, \tau^{\prime}\right)\right]_{x^{\prime}=\mathrm{const} .} d S
$$

where the $g_{i}$ are appropriate functions (products of $v$ with coefficients of $L^{\prime}$ ) and the $S_{i}$ are appropriate faces of $E^{\prime}$, but not the faces $x_{n}^{\prime}=0$ and $t^{\prime}=0$. Noting that $\partial^{3} G^{\prime} / \partial x^{\prime} \partial \xi^{2}, \partial^{3} G^{\prime} / \partial x^{\prime} \partial \tau^{\prime}$ and $\partial^{3} G^{\prime} / \partial x^{\prime} \partial \xi^{\prime} \partial \tau^{\prime}$ are continuous functions provided $\left|x^{\prime}-\xi^{\prime}\right|^{2}+\left|t^{\prime}-\tau^{\prime}\right|>0$, the proof of (1.29) is easily completed.

1.2.5. Conclusion. From the maximum principle for parabolic equations [9] it follows that

$$
\underset{D}{\text { l.u.b. }}|v| \leqq A_{21} \underset{D}{1 . u . b .}|\tilde{f}|
$$

Using (1.33) in (1.32) we get

$$
|v|_{1+\alpha} \leqq K \underset{D}{\text { l.u.b. }}|f(x, t, u, \nabla u)| \quad\left(u=u(x, t) \varepsilon C_{M}\right)
$$

where $K$ depends only on $L$ and $D$.

To complete the proof of (1.7) we shall have to make the following assumption on $f$ :

(C) There exists a positive constant $M$ such that

$$
K|f(x, t, u, \nabla u)| \leqq M
$$

holds for all $(x, t) \varepsilon \bar{D}$ and $u=u(x, t) \varepsilon C_{M}$ (that is, $\left.|u|_{1+\alpha} \leqq M\right)$ where $K$ is the constant of (1.34) (which depends only on $L$ and $D$ ). 
From (1.34) we conclude: If $f$ satisfies Property (C) then (1.7) holds and, consequently, $T$ maps $C_{M}$ into itself.

We now observe that the proof of (1.34) holds for any $\alpha<1$. Taking, in particular, $\beta$ such that $\alpha<\beta<1$ we have

$$
|v|_{1+\beta} \leqq \text { const. l.u.b. }|f(x, t, u, \nabla u)| \leqq K^{\prime} \quad(v=T u)
$$

where $K^{\prime}$ is independent of the specific $u$ in $C_{M}$. Hence $T$ maps $C_{M}$ into a bounded set in $C_{1+\beta}$. Since bounded sets in the norm ||$_{1+\beta}$ are precompact in the norm ||$_{1+\alpha}$, we have proved that $T$ maps $C_{M}$ into a precompact subset of $C_{M}$.

1.3. Existence of solutions. Having proved that $T$ maps $C_{M}$ into a precompact subset of $C_{M}$ we now note that $C_{M}$ is a closed bounded convex set in the Banach space $C_{1+\alpha}$. We further note that $T$ is a continuous transformation (if $\left|u-u_{m}\right|_{1+\alpha} \rightarrow 0$ then $\left|T u-T u_{m}\right|_{1+\alpha} \rightarrow 0$ ) and therefore also completely continuous. Hence we can apply SCHAUDER's fixed point theorem [12] and thus conclude that there exists a function $u$ in $C_{M}$ for which $u=T u$. Also $u \varepsilon C_{2+\delta}$, $L u=f(x, t, u, \nabla u)$ and $u$ vanishes on $\partial D$; the proof of the existence of a solution is thus completed. We have established the following theorem:

Theorem 1. Let $L$ satisfy the assumptions (A), (B) and let the lateral boundary $C$ of $D$ satisfy the assumption $\left(\mathrm{E}^{\prime}\right)$. Let $f$ be defined in the cylinder (3) and satisfy a Hölder condition in compact subsets of it. If $f$ satisfies Property (C) then there exists in $D$ a solution of equation (4) which vanishes on $\partial D=B+C$ ( $B$ is the basis of D); moreover, for some $\delta>0,|u|_{2+\delta}<\infty$.

1.3.1. Examples of functions satisfying Property (C). From Theorem 1 it follows that it is desirable to find simple conditions on $f$ under which Property (C) is satisfied. We shall assume that $f$ satisfies the inequality

$$
|f(x, t, u, w)| \leqq A+B|u|^{\lambda}+C|w|^{\mu},
$$

where $w$ is an $n$-dimensional variable, and find conditions on the positive constants $A, B, C, \lambda, \mu$ under which Property (C) holds.

Case (i): $\lambda<1, \mu<1$.

We claim that for any constants $A, B, C$ Property (C) is satisfied. Indeed, from (1.35), (1.36) it follows that all we need to show is that

$$
K A+K B M^{\lambda}+K C M^{\mu} \leqq M
$$

holds for some positive $M$; clearly this is the case if $M$ is sufficiently large.

Case (ii): $\lambda, \mu, A$ arbitrary.

We claim that if $B$ and $C$ are sufficiently small then Property (C) is satisfied. Indeed, take in (1.37) $M=2 K A$. Then (1.37) is reduced to

$$
B M^{\lambda}+C M^{\mu} \leqq A
$$

which is clearly satisfied for small $B$ and $C$. 
One can easily write down other conditions under which (1.37) is satisfied. We remark that a careful examination of the estimates which led to (1.34) shows that if we replace $D$ by $D_{\tau}$ then $K$ depends on $\tau$ and $K / \tau^{\beta} \rightarrow 0$ when $\tau \rightarrow 0$ for any positive $\beta$ smaller than $\frac{1}{2}$, provided $\alpha$ is taken to be sufficiently small $(2 \beta<1-\alpha)$. Hence, for any constants $A, B, C, \lambda, \mu,(1.37)$ is satisfied in $D_{\tau}$ provided $\tau$ is sufficiently small. Thus if $f$ satisfies (1.36) with any constants $A$, $B, C, \lambda, \mu$ then there exists a solution of the Dirichlet problem

$$
\text { Lu }=f(x, t, u, \nabla u) \text { in } D_{\tau}, \quad u=0 \text { on } \partial D_{\tau},
$$

provided $\tau$ is sufficiently small.

In the next section we shall replace Property (C) by a different property and then find other simple conditions on $f$ under which there exists a solution of the Dirichlet problem.

1.4. Remarks and extensions. If $D$ is a rectangular domain then Theorem 1 can be proved without referring to the results of [7]. Indeed, we then define the transformation $v=T u$ in the following way:

$$
v(\xi, \tau)=\iint_{D_{r}} G(x, t ; \xi, \tau) f(x, t, u(x, t), \nabla u(x, t)) d x d t
$$

where $G$ is Green's function in the domain $D$. This transformation we define on the set $F_{M}$ of functions $u(x, t)$ with finite norms

$$
|u|_{1}=\underset{D}{\operatorname{lou} . b .}(|u|+|\nabla u|) \leqq M
$$

and prove that, for any positive $\alpha<1$,

$$
|v|_{1+\alpha} \leqq \text { const. 1.u.b. }|f| .
$$

Then by Schauder's fixed point theorem [12] it follows that there exists a function $u$ in $F_{M}$ for which $u=T u$. If $f$ is Hölder continuous in compact subsets of the cylinder (3), then it follows (on using the explicit formula for $G$ and [4; Theorems $1,2]$ ) that $u$ is a solution of (4). From [8] we also conclude that $u$ vanishes on $\partial D$.

We remark that in deriving (1.41) we find that the constant which appears on the right side can be taken to be of the form $K|D|^{\gamma}$ where $K$ depends on $\gamma, L$ and the diameter $|B|$ of the basis $B,|D|$ is the diameter of $D$ and $\gamma$ is any positive constant smaller than $\frac{1}{2}$ (see also [10], [11]).

1.4.1. Weak solutions. If we consider the fixed points of the transformation $v=T u$ defined by (1.39) as weak solutions of the Dirichlet problem, then in order to prove the existence of weak solutions it is sufficient to show that the set $F_{M}$ defined by (1.40) is mapped by $T$ into a set of functions in $F_{M}$ which are equally continuous together with their first $x_{i}$-derivatives. (By ArzelA's theorem, such a set is precompact in the norm ||$\left._{1}.\right)$

To prove the equal-continuity of the set $\left\{T u \mid u \varepsilon F_{M}\right\}$ we need to make 
use only of (1.10) with $i, j=0,1$. The proofs of these inequalities follow from [8] under the assumptions $\left(\mathrm{A}^{\prime}\right),(\mathrm{B})$ on $L$; assumption $\left(\mathrm{A}^{\prime}\right)$ is defined as follows:

(A') The functions

$$
a_{i j}, \frac{\partial}{\partial x_{\lambda}} a_{i j}, \frac{\partial^{2}}{\partial x_{\lambda} \partial x_{\mu}} a_{i j}, \frac{\partial}{\partial t} a_{i j}, b_{i}, \frac{\partial}{\partial x_{\lambda}} b_{i}, c
$$

are Hölder continuous in $\bar{D}$.

We have thus proved: If $D$ is a rectangular domain and if the assumptions. $\left(\mathrm{A}^{\prime}\right),(\mathrm{B}),(\mathrm{C})$ hold and $f$ is continuous in the cylinder (3), then there exists a weak. solution $u(x, t)$ of the equation (4) which vanishes on the boundary $\partial D$.

The last part of the theorem (that $u=0$ on $\partial D$ ) follows from [8].

1.4.2. The case $f=f(x, t, u)$. If $f=f(x, t, u)$ and $D$ is a general domain as in Theorem 1, then to prove existence of solutions (in the usual sense) we can start with $C_{M}$ defined in the following way:

$$
C_{M}=\left\{\left.u|| u\right|_{\alpha} \leqq M\right\}
$$

We then need to make use only of (1.10) with $i+j \leqq 3$ and (1.11) with $i+j \leqq 1^{\text {* }}$ Consequently, we can replace the assumptions $(\mathrm{A}),\left(\mathrm{E}^{\prime}\right)$ by weaker assumptions. It is enough, for instance, to assume that $a_{i j}$ have four continuous derivatives in $\bar{D}, b_{i}$ and $c$ have continuous second derivatives in $\bar{D}$ and the functions $h$ (in the local representation of $C$ ) have five continuous derivatives in all their arguments.

\section{Uniqueness Theorems. Another Existence Theorem}

In this section, unless the contrary is explicitly stated, $f(x, t, u, w)$ ( $w$ is $n$-dimensional) is assumed to be merely continuous in the cylinder (3), the coefficients of $L$ are assumed to be merely continuous in $\bar{D}$ and the coefficient $c(x, t)$ of $u$ in $L$ is assumed to be non-positive. A function $v(x, t)$ defined in $\bar{D}$ is said to be regular if it is continuous in $\bar{D}$ and if the functions

$$
\frac{\partial v}{\partial x_{\lambda}}, \quad \frac{\partial^{2} v}{\partial x_{\lambda} \partial x_{\mu}}, \quad \frac{\partial v}{\partial t}
$$

are continuous in $D$. Unless the contrary is explicitly stated, all functions in this section are assumed to be regular.

We shall now prove a few simple theorems concerned with uniqueness of solutions of the equation $L u=f$. Later on we shall combine some of these theorems with Theorem 1 and obtain a new existence theorem (Theorem 2).

2.1. Let the functions $u, \bar{u}$ satisfy $L u=f, L \bar{u}=\bar{f}$ where $\bar{f}$ satisfies the same properties as $f$, and assume that

$$
f(x, t, u, w)>\bar{f}(x, t, \bar{u}, w) \text { in } D \text { whenever } \bar{u}<u \text {. }
$$

If $\bar{u} \geqq u(\bar{u}>u)$ on $\partial D$, then $\bar{u} \geqq u(\bar{u}>u)$ in $D$. 
Proof. It will be enough to consider the case $\bar{u} \geqq u$ on $\partial D$. If the assertion does not hold, then $v=\bar{u}-u$ assumes its negative minimum at an interior point $\left(x^{0}, t^{0}\right)$ of $D$. At this point $\nabla u_{x}=\nabla \bar{u}_{x}$ and $\bar{u}<u$; consequently,

$$
L v\left(x^{0}, t^{0}\right)=\bar{f}\left(x^{0}, t^{0}, \bar{u}\left(x^{0}, t^{0}\right), \nabla u\left(x^{0}, t^{0}\right)\right)-f\left(x^{0}, t^{0}, u\left(x^{0}, t^{0}\right), \nabla u\left(x^{0}, t^{0}\right)\right)<0
$$

which involves a contradiction since $L v \geqq 0$ at every interior point where $v$ assumes its negative minimum.

2.2. Let $\bar{u}, \underline{u}$ and $u$ satisfy the following properties: $L \bar{u}<f(x, t, u, \nabla \bar{u})$ whenever $\bar{u}\langle u, L \underline{u}\rangle f(x, t, u, \nabla \underline{u})$ whenever $\underline{u}>u$ and $L u=f(x, t, u, \nabla u)$. If $\bar{u} \geqq$ $u \geqq \underline{u}(\bar{u}>u>\underline{u})$ on $\partial D$, then $\bar{u} \geqq u \geqq \underline{u}(\bar{u}>u>\underline{u})$ in $D$.

The proof is similar to that of 2.1 .

2.3. Let $f(x, t, u, 0)$ be monotone increasing in $u$ and let $f(x, t, 0,0)=0$. Then zero is the only solution of the equation $L u=f(x, t, u, \nabla u)$ which vanishes on $\partial D$.

Proof. Apply 2.2 with $\underline{u}=0$ and with $\bar{u}=0$.

2.4. Assume that $f(x, t, u, w)<f(x, t, \bar{u}, w)$ whenever $u<\bar{u}$. Then there exists at most one solution of the Dirichlet problem Lu $=f$ with non-homogeneous boundary values $u=u_{0}$ on $\partial D$.

Proof. Suppose there exist two solutions $u^{1}$ and $u^{2}$ and let $u=u^{1}-u^{2}$. If $u \neq 0$ then we may assume that $u$ takes its positive maximum at an interior point $\left(x^{0}, t^{0}\right)$ of $D$ (the case of a negative minimum can be treated in the same way). Since $\nabla u^{1}=\nabla u^{2}$ and $u^{1}>u^{2}$ at $\left(x^{0}, t^{0}\right)$ we get

$$
\begin{aligned}
& L u\left(x^{0}, t^{0}\right)=f\left(x^{0}, t^{0}, u^{1}\left(x^{0}, t^{0}\right), \nabla u^{1}\left(x^{0}, t^{0}\right)\right) \\
&-f\left(x^{0}, t^{0}, u^{2}\left(x^{0}, t^{0}\right), \nabla u^{1}\left(x^{0}, t^{0}\right)\right)>0 .
\end{aligned}
$$

On the other hand, we have $L u \leqq 0$ at every interior point where $u$ takes its positive maximum and we have thus arrived at a contradiction.

2.5. The result of 2.4 is valid under the weaker assumption that $f(x, t, u, w) \leqq$ $f(x, t, \bar{u}, w)$ whenever $u<\bar{u}$.

Proof. The transformation $v=e^{-\alpha t} u(\alpha>0)$ transforms the equation $L u=f$ into $L v=\tilde{f}$ where $\tilde{f} \equiv f e^{-\alpha t}+\alpha v$. We can now apply 2.4 to $v$ and thus conclude that $v$ (and therefore $u$ ) is uniquely determined from its boundary values on $\partial D$.

2.6. If for some constant $M$

$$
|f(x, t, u, w)-f(x, t, \vec{u}, w)| \leqq M|u-\bar{u}|,
$$

then there exists at most one solution of $L u=f$ with prescribed boundary values on $\partial D$.

Proof. The function $v=u e^{-B t}$ satisfies

$$
L v=f(x, t, u, \nabla u) e^{-B t}+B v \equiv \tilde{f}(x, t, v, \nabla v) .
$$


If we take $B>M$ then our assertion follows from 2.4 .

2.7. Let $\underline{w}, \bar{w}$ be functions which satisfy

$$
\underline{w}(x, t)<0<\bar{w}(x, t) \text { in } \bar{D} .
$$

Let $\tilde{C}$ be the set of points $(x, t, u, w)$ which satisfy

$$
(x, t) \varepsilon \bar{D}, \quad \underline{w}(x, t) \leqq u \leqq \bar{w}(x, t), \quad-\infty<w_{i}<\infty \quad(i=1, \cdots, n),
$$

where $w=\left(w_{1}, \cdots, w_{n}\right)$.

If $L$ satisfies (A), (B) and $C$ satisfies $\left(\mathrm{E}^{\prime}\right)$, if $f$ is Hölder continuous in the cylinder (3) and satisfies Property (C), and if

$$
L w>f(x, t, \underline{w}, \nabla w), L \bar{w}<f(x, t, \bar{w}, \nabla \bar{w})
$$

then the Dirichlet problem $L u=f$ in $D, u=0$ on $\partial D$, has a solution in $D$ which satisfies the inequality

$$
w(x, t) \leqq u(x, t) \leqq \bar{w}(x, t) \text { in } D .
$$

Proof. Define $F(x, t, u, w)=f\left(x, t, u^{\prime}, w\right)$ where $u^{\prime}$ is defined for every $(x, t)$ in $\bar{D}$ in the following way:

$$
u^{\prime}=\left\{\begin{array}{lll}
w(x, t) & \text { if } \quad u<w(x, t) \\
u & \text { if } \quad \underline{w}(x, t) \leqq u \leqq \bar{w}(x, t) \\
\bar{w}(x, t) & \text { if } \quad u>\bar{w}(x, t) .
\end{array}\right.
$$

It is elementary to show that, together with $f, F$ is also Hölder continuous. Furthermore,

$$
\begin{array}{lll}
L \underline{w}>F(x, t, u, \nabla w) & \text { whenever } & u<w(x, y), \\
L \bar{w}<F(x, t, u, \nabla \bar{w}) & \text { whenever } u>\bar{w}(x, y) .
\end{array}
$$

Now, since $f$ satisfies Property (C), also $F$ satisfies it and, consequently, there exists a solution of $L u=F(x, t, u, \nabla u)$ which vanishes on $\partial D$. (2.4) is a consequence of 2.2 and (2.5).

\subsection{An existence theorem.}

Theorem 2. Let $L$ satisfy the assumptions (A), (B) and let $C$ satisfy condition $\left(\mathrm{E}^{\prime}\right)$. Assume that there exist two regular functions $w$ and $\bar{w}$ satisfying (2.1) and (2.3), and let $f(x, t, u, w)$ be bounded in the domain $\widetilde{C}$ defined by (2.2) and Hölder continuous in compact subsets of $\tilde{C}$. Then there exists a solution $u(x, t)$ in $D$ of the equation (4) which vanishes on $\partial D$; $u$ satisfies (2.4) and, for some positive $\delta$, $|u|_{2+\delta}<\infty$.

Proof. The proof follows from 2.7 by noting that the function $F$ constructed in 2.7 satisfies Property (C). 
We remark that Theorem 2 remains true if the assumption that $c(x, t) \leqq 0$ is removed. Indeed, a transformation of the form $v=u e^{\beta t}$ reduces the case of general $c$ to the case of non-positive $c$.

2.9. Examples of functions $f$ for which Theorem 2 is valid. It will be enough to consider the case $c(x, t) \leqq 0$. Assume that $f$ satisfies the inequality

$$
|f(x, t, u, w)| \leqq A+B|u|^{\lambda}
$$

in the cylinder (3). Take

$$
\bar{w}(x, t)=\varphi(t)>0, \quad \underline{w}(x, t)=-\varphi(t)<0 .
$$

Then

$$
L \bar{w}=c \varphi-\varphi^{\prime} \leqq-\varphi^{\prime}, \quad L \underline{w}=-c \varphi+\varphi^{\prime} \geqq \varphi^{\prime} .
$$

Hence (2.3) is valid if $\varphi$ satisfies

$$
\varphi^{\prime}(t)-B[\varphi(t)]^{\lambda}-A>0 .
$$

Clearly, if (2.8) holds then, in the domain $\tilde{C}$ (defined in terms of $\varphi(t)$ ), $f$ will be bounded and, consequently, Theorem 2 is valid.

Case 1: $\lambda<1$.

If we take $\varphi(t)=H(t+1)$ then (2.8) becomes

$$
H-B H^{\lambda}(t+1)^{\lambda}-A>0
$$

which is clearly satisfied for any $A, B$ if $H$ is sufficiently large.

Case 2: $\lambda=1$.

(2.8) is satisfied by any positive solution of the equation

$$
\varphi^{\prime}(t)-B \varphi(t)-A^{\prime}=0
$$

where $A^{\prime}$ is any fixed number larger than $A$. For $H$ positive and sufficiently large

$$
\varphi(t)=H e^{B t}-\frac{A^{\prime}}{B}
$$

is a positive solution of $(2.9)$.

Case 3: $\lambda>1$.

Taking $\varphi(t)=H(t+\epsilon)$ where $\epsilon>0$, (2.8) is reduced to

$$
H-B H^{\lambda} b^{\lambda}-A>0
$$

(since $\epsilon$ may be taken arbitrarily small). Here $b$ is the height of $D$. Taking $H=2 A$ we reduce (2.10) to

$$
A>B(2 A)^{\lambda} b^{\lambda}
$$


Hence (2.8) is valid provided

$$
b<\frac{1}{2}\left(\frac{A^{1-\lambda}}{B}\right)^{1 / \lambda} .
$$

Note that Case 1 is included in $\S 1$, Case 2 is not included in $\$ 1$ and Case 3 gives a more precise condition than the condition which one can derive from $\$ 1$ when $\mu=0$. Case 1 was also considered by PINI [10] using different functions for $\bar{w}$ and $w$.

2.10. Non-homogeneous boundary values. In Theorems 1,2 we proved the existence of solutions of the Dirichlet problem $L u=f$ with vanishing boundary values. To discuss the case of the general boundary conditions (5) we assume that there exists a function $\psi(x, t)$ having second $x$-derivatives and one $t$-derivative all of which are Hölder continuous in $\bar{D}$ and such that $\psi=g$ on $B$ and $\psi=k$ on $C$. We then can prove the existence of a solution of the problem (4), (5).

Indeed, writing $v=u-\psi$, our problem is reduced to that of finding a solution of the equation

$$
L v=f(x, t, v+\psi, \nabla v+\nabla \psi)-L \psi \equiv \tilde{f}(x, t, v, \nabla v)
$$

with zero boundary values. Since $\tilde{f}$ is Hölder continuous in all its arguments in the cylinder (3), we have reduced our present problem to the one considered in Theorems 1, 2.

2.11. Uniqueness. Theorems 1,2 consider the problem of existence of solutions. We recall that the question of the uniqueness of solutions was considered in 2.4, 2.5 and 2.6. One can easily formulate conditions on $f$ such that the problem (4), (5) has one and only one solution.

\section{Elliptic Equations}

The results of $\S \S 1,2$ can be extended to second order elliptic equations. Writing such an equation in the form

$$
\bar{L} u=\sum_{i, j=1}^{n} a_{i j}(x) \frac{\partial^{2} u}{\partial x_{i} \partial x_{i}}+\sum_{i=1}^{n} b_{i}(x) \frac{\partial u}{\partial x_{i}}+c(x) u=f(x, u, \nabla u),
$$

we make the following assumptions:

(i) The coefficients of $\bar{L}$ are defined and Hölder continuous (exponent $\lambda$ ) in the closure $\bar{B}$ of a bounded domain $B ; c(x) \leqq 0$;

(ii) $\bar{L}$ is elliptic at each point of $\bar{B}$;

(iii) For each point $P$ on $\partial B$ there exists a neighborhood $V$ such that $V \cap \partial B$ can be written, for some $i$, in the form

$$
x_{i}=h\left(x_{1}, \cdots, x_{i-1}, x_{i+1}, \cdots, x_{n}\right)
$$

where $h$ has Hölder continuous (exponent $\sigma>\lambda$ ) second $x$-derivatives. 
Under conditions weaker than the above ones, Condes [1; p. 307] (for $n>2$ ) and Bers \& NIRENBERg [0; pp. 6-7] (for $n=2$ ) have proved that if $u$ is a solution of the equation $L u=g(x)$ in $B$, which has second continuous $x$-derivatives in $\bar{B}$ and which vanishes on $\partial B$, then there exists an $\alpha>0$ depending only on $L$ and $B$ such that

$$
|u|_{1+\alpha} \leqq K \underset{B}{\operatorname{lou} . b .}|g|
$$

where $K$ depends only on $L$ and $B$ (not on $g$ ) and where

$$
|u|_{1+\alpha}=\underset{B}{1 . u . b .}(|u|+|\nabla u|)+\underset{B}{1 . u . b .} \frac{|\nabla u(x)-\nabla u(y)|}{|x-y|^{\alpha}}
$$

If we use this result, together with the Schauder theory, then we can extend Theorem 1 (and then also Theorem 2) to elliptic equations; moreover, the assumptions on $L$ and $B$ are weaker than those made in the analogous parabolic case. In particular, we have the following theorem:

Assume that (i), (ii), (iii) hold. Let $f(x, u, \nabla u)$ be defined in the cylinder

$$
x \varepsilon \bar{B}, \quad-\infty<u, u_{x_{i}}<\infty,
$$

and be Hölder continuous in compact subsets of it. Let the inequality

$$
|f(x, u, \nabla u)| \leqq A+B|u|^{\beta}+C|u|^{\gamma}
$$

be satisfied for some non-negative constants $A, B, C, \beta, \gamma$. There exists a solution of the equation (3.1) which vanishes on $\partial B$, provided one of the following two conditions hold:

(a) $A, B, C$ are arbitrary; $\beta<1, \gamma<1$;

(b) $A, \beta, \gamma$ are arbitrary; $B$ and $C$ are sufficiently small. Moreover, for some $\delta>0,|u|_{1+\delta}+|\nabla u|_{1+\delta}<\infty$.

Added in proof. When we prepared Sections 1, 2 of this paper, we were not familiar with the estimate of Condes [1] mentioned above. We plan to try to prove an analogous estimate for parabolic equations. With such an estimate at hand, Theorems 1,2 will hold under very weak assumptions on $L$ and $D$, analogous to the assumptions (i), (ii), (iii) of the present section.

\section{REFERENCES}

[0] L. Bers \& L. Nirenberg, On linear and nonlinear elliptic boundary value problems in the plane, Convegno Internazionale sulle Equazioni Derivate e Parziali, Trieste (1954), pp. 141-167.

[1] H. O. CoRdes, Über die erste Randwertaufgabe bei quasilinearen Differentialgleichungen zweiter Ordnung in mehr als zwei Variablen, Math. Ann., 131 (1956), pp. 278-312.

[2] C. Ciliberto, Su di un problema al contorno per una equazione non lineare di tipo parabolico in due variabili, Ricerche Mat., 1 (1952), pp. 55-77. 
[3] C. Ciniberto, Su di un problema al contorno per l'equazione $u_{x x}-u_{y}=f\left(x, y, u, u_{x}\right)$, Ricerche Mat., 1 (1952), pp. 295-316.

[4] F. G. Dressel, The fundamental solution of the parabolic equation, Duke Math., J., 7 (1940), pp. 186-203.

[5] C. D. Eidelman, On fundamental solutions of parabolic systems, Math. Sbornik (N.S.), 38 (80) (1950), pp. 51-92.

[6] A. Friedman, Interior estimates for parabolic systems of partial differential equations, J. Math. and Mech., 7 (1958), pp. 393-417.

[7] A. Friedman, Boundary estimates for second order parabolic equations and their applications, J. Math. and Mech., 7 (1958), pp. 771-791.

[8] A. Friedman, Parabolic equations of the second order, to appear in Trans. Amer. Math. Soc.

[9] L. Nirenberg, A strong maximum principle for parabolic equations, Comm. Pure Appl. Math., 6 (1953), pp. 167-177.

[10] B. Pinr, Sul primo problema di valori al contorno per l'equazione parabolica non lineare del secondo ordine, Rend. Sem. Mat. Univ. Padova, 27 (1957), pp. 149-161.

[11] T. Satō, Sur l'équazion aux derivees partielles $\Delta z=f(x, t, z, p, q)$, Comp. Math., 12 (1954), pp. 157-177.

[12] J. SchaUder, Der Fixpunktsatz in Funktionalraümen, Studia Math., 2 (1930), pp. 171-180.

Indiana University Bloomington, Indiana 